\title{
Therapeutic impact of cholecalciferol in patients with psychiatric disorders receiving olanzapine
}

\author{
Somayeh Kaviyani ${ }^{1 *}$, Mohammad Bahadoram², Gholamreza Houshmand ${ }^{3}$, Samaneh Bahadoram ${ }^{2}$
}

\begin{abstract}
Introduction: Psychiatric disorders such as schizophrenia require appropriate treatment. Second-generation antipsychotics, including olanzapine, are administered as an appropriated treatment for these disorders. However, this drug has several complications, such as obesity and increased waist circumference which reduces the acceptance of the treatment.

Objectives: The aim of this study was to investigate the effects of vitamin D3 therapy on waist circumference caused by olanzapine. Patients and Methods: In this randomized clinical trial, 50 hospitalized women with psychiatric disorders receiving olanzapine were divided into two groups of intervention and control groups. Before the intervention, the waist circumference, hip circumference, and waist-hip ratio were measured. Then, for 12 weeks, the intervention group received vitamin D3 and the control group received paraffin as the placebo, one pearl every 2 weeks. Waist circumference, hip circumference, and waist-hip ratio were re-measured and the collected data were analyzed by pair $t$ test.

Results: Our findings showed that there is a significant decrease in waist circumference and waist-hip ratio in intervention group $(P<0.001)$. However, these changes were not significant in the placebo group. Hip circumference was not changed significantly in both groups.

Conclusion: Vitamin D can be administered concomitant with 0lanzapine to improve side effects of olanzapine such as increasing waist circumference.

Keywords: Olanzapine, Vitamin D3, Waist circumference, Waist-hip ratio, Psychiatric disorders

Please cite this paper as: Kaviyani S, Bahadoram M, Houshmand G, Bahadoram S. Therapeutic impact of cholecalciferol in patients with psychiatric disorders receiving olanzapine. J Parathyr Dis. 2018;6(1):19-22. DOI: 10.15171/jpd.2018.07.

Copyright $\odot 2018$ The Author(s); Published by Nickan Research Institute. This is an open-access article distributed under the terms of the Creative Commons Attribution License, which permits unrestricted use, distribution, and reproduction in any medium, provided the original work is properly cited.
\end{abstract}

\section{Introduction}

Psychiatric disorders, including schizophrenia and mood disorders, require effective and appropriate treatment. Olanzapine is a second-generation antipsychotic drug that has very low-peripheral side effects and is administered to treat various psychiatric disorders including psychosis, obsessive-compulsive or bipolar disorders. This drug is also administered for the treatment of Huntington's disease. Olanzapine has several side effects, including weight gain, increasing blood lipids, and increasing risk of diabetes and cardiovascular disease. Comparing with other second-generation antipsychotic agents, administration of olanzapine leaded to a higher proportion of weight gain and obesity. Olanzapine results in obesity by type 1 histamine receptors (1). The most common forms of olanzapine are 5 and $10 \mathrm{mg}$ pills, administered 10 to 20 $\mathrm{mg} / \mathrm{d}$.

Obesity is the second major health problem (2). Central obesity and a high waist-hip ratio of over 0.9 in females and over one in males are independently associated with a higher risk of metabolic syndrome (1). According to standard definitions, metabolic syndrome is defined as:

1) Abdominal obesity as waist circumference above 102 $\mathrm{cm}$ in males and above $88 \mathrm{~cm}$ in females.

2) Hypertension above $130 / 85 \mathrm{~mm} \mathrm{Hg}$.

3) Triglyceride levels above $150 \mathrm{mg} / \mathrm{dL}$.

4) Fasting blood glucose above $100 \mathrm{mg} / \mathrm{dL}$.

5) HDL cholesterol less than 40 in males and less than 50 in females (3).

Concerning cardiovascular and metabolic factors, higher waist circumference is associated with increased risk of cardiovascular disease (4).

Various modalities were tested to reduce waist circumference and metabolic syndrome caused by administration of olanzapine.

Vitamin D3 is one of the drugs supposed to have association with obesity (5). Cholecalciferol (vitamin D3) is the available form of this vitamin (6) and its oral form is consisted of $50000 \mathrm{IU}$ of vitamin D3 (7). Many obese people have low levels of this vitamin (4). The normal serum level of vitamin $\mathrm{D}$ is 30 to $80 \mathrm{ng} / \mathrm{mL}$ (8). Additionally vitamin D3 is effective in improving psychiatric disorders 
Implication for health policy/practice/research/ medical education

Vitamin D3 supplement therapy can improve side effects of olanzapine such as increasing waist circumference .

such as depression and schizophrenia (9).

Various studies have been conducted to reduce waist circumference and consequently metabolic syndrome caused by olanzapine. Numerous studies have also been conducted on drug-related or non-drug-related obesities. For example, Soares et al conducted a controlled clinical trial on this subject in Australia. The results of their study showed, receiving low serum levels of vitamin D3 was associated with a higher prevalence of obesity and increased waist circumference. It was also found that vitamin D3 was effective in weight loss by increasing fat oxidation after meals (10). Likewise, Song et al obtained similar findings of Soares et al. However, they reported a different mechanism for weight reduction impact of vitamin $\mathrm{D}$. They concluded a role for vitamin D to regulate of fat cell death and lipid metabolism (11). Similarly, Salehpour et al investigated the association between vitamin D deficiency and obesity. They evaluated the effects of 12-week vitamin $\mathrm{D}$ supplementation on anthropometric indexes in obese and overweight women. The results of their study showed an inverse relationship between serum vitamin $\mathrm{D}$ level and body fat mass, including waist circumference and weight. In overweight obese women, vitamin D level was increased by vitamin D3 supplementation and it led to a decrease in body fat (5). Accordingly, Tamer et al assessed the incidence of obesity and abdominal obesity in women without diabetes and without metabolic syndrome. The studied women were divided into two groups based on their vitamin D3 levels. The first group included people with vitamin $\mathrm{D}$ deficiency and the second group included women without vitamin deficiency. They measured body mass index (BMI), waist circumference, and waist-hip ratio and compared them between the two groups. The results showed that BMI, waist circumference, waist-hip ratio, obesity and abdominal obesity was lower in the group without vitamin D deficiency, as compared to the group with vitamin D deficiency. They concluded, vitamin D levels have an inverse relationship with BMI (12).

Mai et al showed that low levels of vitamin D was associated with a higher incidence of obesity. In subjects with a BMI greater than $30 \mathrm{~kg} / \mathrm{m}^{2}$, serum vitamin D levels were less than $50 \mathrm{nmol} / \mathrm{L}$. They found low serum levels of vitamin $\mathrm{D}$ was associated with a significant increase in odd ratios of the incidence of obesity during follow-up. In addition, vitamin D serum levels of less than $50 \mathrm{nmol} / \mathrm{L}$ were clearly associated with obesity with a recent onset in adults (13). Recent investigations have focused on vitamin D deficiency and its association with obesity as an important health issues. Studies have shown that obese people who are suffering from vitamin D3 deficiency may also suffer from poor regulation of adipose tissue. It is possible that adipose tissue may be a direct target for vitamin D3 implication.
Mai et al (13) also highlighted the bioavailability of vitamin D3 receptors and 25-hydroxyvitamin D3 1-alphahydroxylase, in mice and human fat cells. There are some evidences on the effects of vitamin D3 on body fat, through inhibiting the transcription of lipid cells and regulating fat during the differentiation of fat cells. The study by Ding et al showed vitamin D metabolites also affect adiponectin products and inflammatory responses in adipose tissue (14).

Olanzapine is effective in treating all psychiatric disorders. According to the low incidence of off-pyramid side effects, this drug is effectively used for the treatment of patients. However, it has some complications such as obesity and increased waist circumference and is associated with an increased risk of cardiovascular diseases and metabolic syndrome that can reduce the acceptance of treatment by the patients.

\section{Objectives}

The aim of this study was to investigate the effect of vitamin D3 on reducing obesity and overweight caused due to the use of olanzapine in women with psychiatric disorders.

\section{Patients and Methods Study population}

In this clinical trial, we studied 50 hospitalized female patients who were under treatment of olanzapine in psychiatry ward of Ahvaz Golestan hospital from September to March 2014. Patients were divided into two groups of 25 patients for each as control and intervention groups randomly.

Inclusion criteria were: age group between 20 to 25 years old, not having a medical illness, normal serum vitamin D level, absence of pregnancy, and a weight gain of around 3-4 kg after receiving olanzapine. Before the intervention, the waist circumference, hip circumference, and waisthip ratio were measured and recorded. Patients with an increase in the above mentioned indices were considered for a complete medical history, liver and kidney tests assessments. Additionally, their serum levels of vitamin D3 were checked (serum vitamin D3 levels should be above $30 \mathrm{ng} / \mathrm{mL}$ to be an inclusion criteria) (8).

To conduct the study, for 12 weeks vitamin D3 pearl was administered for the intervention group, one pearl per week. In addition, for 12 weeks paraffin pearl as placebo was administered for the control group, one pearl per week. The instructions for the administration of pearls was recommended by nutrition advisors. Both vitamin D3 and paraffin pearls were obtained from pharmaceutical companies. After the intervention, waist circumference, hip circumference, and waist-hip ratio were re-measured and recorded. Before the intervention, the subjects were instructed not to change their lifestyle during the study.

\section{Ethical issues}

The study protocol was approved by Ethical Committee of Ahvaz Jundishapur University of Medical Sciences (\#U- 
93168). The research followed the tenets of the Declaration of Helsinki. Written informed consent was obtained from all patients and remained confidential.

\section{Statistical analysis}

Sample size was calculated by considering a confidence interval of $95 \%$ and a power of $90 \%$, based upon previous studies. Statistical analysis was conducted using SPSS 18 (software SPSS Inc., Chicago, IL, USA). For evaluating the vitamin D3 efficacy and comparison of waist circumference, hip circumference, and waist-hip ratio between the two groups and also within each group before and after the intervention, independent $t$ test and paired $t$ test were used, respectively. $P$ values less than 0.05 were considered statistically significant.

\section{Results}

According to Table 1, no significant difference of waist circumference, hip circumference, and waist-hip ratio between the two groups of intervention and control before the study was detected $(P>0.05)$.

To investigate the impact of vitamin D3 therapy, waist circumference was compared before and after study in intervention group. We found, waist circumference in the intervention group had a statistically significant difference before and after the administration of vitamin D3 $(P<0.001)$ (Table 2$)$. However in control group, no significant changes was seen.

As it is shown in Table 3, hip circumference in the placebo group, the mean value did not change thus it had no $P$ value. The intervention group also had no statistically significant difference of hip circumference before and after the intervention $(P=0.84)$.

The comparison of waist-hip ratio showed a significant decrease before and after administration of vitamin D3. However, there was no significant changes in control group (Table 4).

Discussion

The results of this study showed, waist circumference was

Table 1. Investigating the waist circumference (cm), hip circumference (cm) and waist-hip ratio in two groups of intervention and control before the intervention.

\begin{tabular}{llll}
\hline Variables & Group & Mean \pm SD & P value \\
\hline \multirow{2}{*}{ Waist circumference } & Intervention & $98.60 \pm 13.42$ & \multirow{2}{*}{0.702} \\
& Control & $100.26 \pm 17.37$ & \\
\multirow{2}{*}{ Hip circumference } & Intervention & $108.84 \pm 10.54$ & \multirow{2}{*}{0.119} \\
& Control & $103.60 \pm 13.00$ & \\
Waist-hip ratio & Intervention & $0.916 \pm 0.094$ & \multirow{2}{*}{0.177} \\
& Control & $0.958 \pm 0.123$ & \\
\hline
\end{tabular}

Table 2. Investigating the waist circumference in two groups

\begin{tabular}{llll}
\hline Group & Time & Mean \pm SD & $P$ value \\
\hline \multirow{2}{*}{ Intervention } & Before intervention & $100.26 \pm 17.37$ & \multirow{2}{*}{$<0.001$} \\
& After intervention & $97.80 \pm 17.06$ & \\
\multirow{2}{*}{ Control } & Before intervention & $98.60 \pm 13.42$ & \multirow{2}{*}{0.478} \\
& After intervention & $98.66 \pm 13.50$ & \\
\hline
\end{tabular}

significantly reduced in intervention group, receiving vitamin D3 $(P<0.001)$, while we did not observe impressive changes in waist circumference and waist-hip ratio, as compared with group who received placebo.

The effects of vitamin D3 on the reduction of waist circumference is also reported in a study by Salehpour et al (5). In addition, other studies such as Tamer et al have found that waist circumference and waist-hip ratio have a negative relationship with the serum level of vitamin D3 (12). In the present study, we found, vitamin D3 was effective in reducing waist circumference and waist-hip ratio.

Mai et al assessed the relationship between waist circumference and obesity and vitamin D levels. The results of their investigation showed, people with larger waist circumference had a vitamin $\mathrm{D}$ level of below $50 \mathrm{ng} / \mathrm{L}$, which is below the normal level. In our study, vitamin D3 serum level was normal in the two groups of patients receiving placebo and vitamin D3. Thus vitamin D3 deficiency, as a confounding factor, was excluded. Hence, considering the normal levels of vitamin D3 in the two groups, it is possible that the reduction of waist circumference and the waist-hip ratio was attributed to vitamin D3 therapy. However, this finding is different from the results of the study by Mai et al (13).

Furthermore, the study of Ding et al revealed that vitamin D3 is effective in metabolism and obesity through controlling adipose tissue, while people with vitamin D3 deficiency are more obese (14). In our study, the difference in waist-hip ratio was statistically significant in intervention group $(P<0.001)$, but there was no statistically significant difference in group receiving placebo $(P=0.56)$.

\section{Conclusion}

This study showed, vitamin D3 was effective in reducing waist circumference and waist-hip ratio, and was generally effective in reducing obesity. It was also known that patients receiving olanzapine experience an increased in waist circumference, metabolic syndrome, and obesity. These problems reduce patients' tendency to accept olanzapine while it is an effective treatment for psychiatric

Table 3. Investigating the hip circumference in two groups

\begin{tabular}{llll}
\hline Group & Time & Mean \pm SD & P value \\
\hline \multirow{2}{*}{ Intervention } & Before intervention & $108.84 \pm 10.54$ & \multirow{2}{*}{0.84} \\
& After intervention & $108.80 \pm 10.60$ & \\
\multirow{2}{*}{ Control } & Before intervention & $103.60 \pm 13.00$ & \multirow{N}{*}{ NA } \\
& After intervention & $103.60 \pm 13.022$ & \\
\hline
\end{tabular}

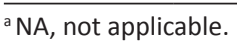

Table 4. Investigating the waist-hip ratio in two groups

\begin{tabular}{llll}
\hline Group & Time & Mean \pm SD & $P$ value \\
\hline \multirow{2}{*}{ Intervention } & Before intervention & $0.916 \pm 0.094$ & \\
& After intervention & $0.894 \pm 0.09$ & $<0.001$ \\
\multirow{2}{*}{ Control } & Before intervention & $0.958 \pm 0.123$ & \\
& After intervention & $0.992 \pm 0.120$ & 0.560 \\
\hline
\end{tabular}

aA, not applicable. 
disorders. The positive effect of vitamin D3, as observed in this study, is improving patient's self-confidence and improved their interpersonal relationships and their adherence to treatments. On the other hand, vitamin D3 is also effective in improving psychiatric disorders, including schizophrenia and depression. Therefore, it is recommended to administer vitamin $\mathrm{D}$ as a supplement, along with psychiatric drugs including olanzapine.

\section{Limitations of the study}

Given the small number of samples and the short duration of the intervention, it is necessary to conduct longer studies with more samples. It is also necessary to conduct further studies on the effects of vitamin D3 and other drugs which have a positive effect on waist circumference and waist-hip ratio and metabolic syndrome caused by the administration of olanzapine (based on other criteria).

\section{Acknowledgments}

This study was part of residential thesis of Dr. Somayeh Kaviyani in Psychiatry. We would like to express our thanks to all the professors and all the patients who participated in the project.

\section{Authors' contribution}

All authors passed four criteria for authorship contribution based on recommendations of the International Committee of Medical Journal Editors.

\section{Conflicts of interest}

The authors declare that they do not have any conflicts of interest.

\section{Funding/Support}

This research was funded by Ahvaz Jundishapur University of Medical Sciences (\# U-93168).

\section{References}

1. Hu S, Yao M, Peterson BS, Xu D, Hu J, Tang J, et al. A randomized, 12-week study of the effects of extendedrelease paliperidone (paliperidone ER) and olanzapine on metabolic profile, weight, insulin resistance, and $\beta$-cell function in schizophrenic patients. Psychopharmacology (Berl). 2013;230:3-13. doi: 10.1007/s00213-013-3073-1.

2. Popović I, Ravanić D, Janković S, Milovanović D, Folić M, Stanojević A, et al. Long-term treatment with olanzapine in hospital conditions: prevalence and predictors of the metabolic syndrome. Srp Arh Celok Lek. 2015;143:712-8.
3. Yoon H, Jeong DK, Park CE, Oh HJ, Kim SG. The association between gender difference with metabolic syndrome, metabolic syndrome score and serum vitamin D levels in Korean adults. Int J Food Sci Nutr. 2017;68:121-9. doi: 10.1080/09637486.2016.1221899.

4. Boumaiza I, Omezzine A, Rejeb J, Rebhi L, Rejeb NB, Nabli $\mathrm{N}$, et al. Association between eight adiponectin polymorphisms, obesity, and metabolic syndrome parameters in Tunisian volunteers. Metab Syndr Relat Disord. 2011;9:419-26. doi: 10.1089/met.2011.0035.

5. Salehpour A, Hosseinpanah F, Shidfar F, Vafa M, Razaghi M, Dehghani S, et al. A 12-week double-blind randomized clinical trial of vitamin $\mathrm{D}_{3}$ supplementation on body fat mass in healthy overweight and obese women. Nutr J. 2012; 11:78. doi: 10.1186/1475-2891-11-78.

6. Nimitphong H, Samittarucksa R, Saetung S, Bhirommuang N, Chailurkit LO, Ongphiphadhanakul B. The effect of vitamin D supplementation on metabolic phenotypes in thais with prediabetes. J Med Assoc Thai. 2015;98:1169-78.

7. Sadiya A, Ahmed SM, Carlsson M, Tesfa Y, George M, Ali SH, et al. Vitamin D3 supplementation and body composition in persons with obesity and type 2 diabetes in the UAE: a randomized controlled double-blinded clinical trial. Clin Nutr. 2016; 35:77-82. doi: 10.1016/j. clnu.2015.02.017.

8. Nguyen $\mathrm{HB}$, Eshete B, Lau KH, Sai A, Villarin M, Baylink D. Serum 1,25-dihydroxyvitamin D: an outcome prognosticator in human sepsis. PLoS One. 2013; 8:e64348. doi: 10.1371/journal.pone.0064348.

9. Schneider B, Weber B, Frensch A, Stein J, Fritz J. Vitamin $\mathrm{D}$ in schizophrenia, major depression and alcoholism. J Neural Transm (Vienna). 2000; 107:839-42.

10. Soares MJ, Murhadi LL, Kurpad AV, Chan She Ping-Delfos WL, Piers LS. Mechanistic roles for calcium and vitamin D in the regulation of body weight. Obes Rev. 2012;13:592605. doi: 10.1111/j.1467-789X.2012.00986.x.

11. Song Q, Sergeev IN. Calcium and vitamin D in obesity. Nutr Res Rev. 2012;25:130-41. doi: 10.1017/S0954422412000029.

12. Tamer G, Mesci B, Tamer I, Kilic D, Arik S. Is vitamin D deficiency an independent risk factor for obesity and abdominal obesity in women? Endokrynol Pol. 2012;63:196-201.

13. Mai XM, Chen Y, Camargo CA Jr, Langhammer A. Cross-sectional and prospective cohort study of serum 25-hydroxyvitamin D level and obesity in adults: the HUNT study. Am J Epidemiol. 2012;175:1029-36. doi: 10.1093/aje/ kwr456.

14. Ding C, Gao D, Wilding J, Trayhurn P, Bing C. Vitamin D signalling in adipose tissue. Br J Nutr. 2012;108:1915-23. doi: $10.1017 /$ S0007114512003285. 